\title{
Crecimiento y fenología de Solanum sessiliflorum Dunal o topiro bajo condiciones agroclimáticas del corregimiento de Vuelta Mansa, municipio de Atrato, Chocó, Colombia
}

\section{Growth and phenology of Solanum sessiliflorum Dunal or topiro under agro climatic conditions of the return corregimiento Mansa, Municipality of Atrato, Department of Chocó, Colombia}

\author{
Haidyn Luis Moreno Mosquera ${ }^{1}$, Samia Yisela Mosquera²
}

\section{Resumen}

Se evaluó el crecimiento y fenología de plántulas de lulo Solanum sessiliflorum Dunal o topiro producida en vivero y trasplantadas a sitio definitivo a $15 \mathrm{~cm}$ de altura. Se empleó un diseño completamente al azar con cuatro tratamientos, tres repeticiones y cuatro réplicas: $T^{l}$ siembra de las plántulas directamente en el suelo, este tratamiento recibió el nombre de testigo; $T^{2}$ siembra de plántulas con aplicación de $200 \mathrm{~g}$ de cal agrícola; $T^{3}$ aplicación de $100 \mathrm{~g}$ de cal agrícola más $400 \mathrm{~g}$ de tierra de hormiga; $T^{4}$ aplicación $400 \mathrm{~g}$ de tierra de hormiga. Los resultados se analizaron mediante el paquete estadístico Statgraphics Centurión versión 2009. Las variables evaluadas fueron: altura, diámetro, número de flores, número de frutos, rendimiento productivo, además de los tiempos de germinación, floración y fructificación. En el experimento se aplicó prueba de ANOVA simple donde no se encontraron diferencia estadisticamente significativa entre los tratamientos para las variables de crecimiento en altura y diámetro $(p>0,85) y$ $(p>0,99)$ respectivamente; también se aplicó las correlaciones momento producto de Pearson donde se identificó una fuerza de relación lineal directa entre el número de flores, masculina $y$ femeninas con el número de frutos ( $p>0,93)$, logrando una producción de 16,3 ton/ha. Se concluyó que después de producir $S$. sessiliflorum en vivero, se reduce la necesidad de adicionar enmiendas al suelo; no obstante, es muy importantela selección de sitios ricos en contenido orgánico y realizar prácticas de manejo como limpias, tutorado y poda que son determinantes para optimizar los rendimientos y productividad.

Palabras clave: Chocó, Colombia, Fenología, Topiro, Vuelta Mansa.

\author{
Investigador asociado tipo 1, Instituto de \\ Investigaciones Ambientales del Pacífico \\ (IIAP), Quibdó, Colombia. \\ e-mail:hmoreno@iiap.org.co \\ 2 Investigadora asociada tipo 2, Instituto de \\ Investigaciones Ambientales del Pacífico \\ (IIAP), Quibdó, Colombia.
}




\section{Bioetnia Volumen 14, 2017}

\section{Abstract}

In this study the growth and phenology of seedlings of lulo Solanum sessiliflorum Dunal or topiro produced in nursery and transplanted to final site at $15 \mathrm{~cm}$ height were evaluated. For which a completely randomized design was used with four treatments, three replications and four replicates: $T^{l}$ planting the seedlings directly in the soil, this treatment was called a witness; $T^{2}$ planting of seedlings with application of $200 \mathrm{~g}$ of agricultural lime; $T^{3}$ application of $100 \mathrm{~g}$ of agricultural lime plus $400 \mathrm{~g}$ of ant soil; $T^{4}$ application $400 \mathrm{~g}$ of ant soil. The results were analyzed using the statistical package Statgraphics Centurion version 2009. The variables evaluated were height, diameter, number of flowers, number of fruits, productive yield in addition to the times of germination, flowering and fruiting. In the experiment, a simple ANOVA test was applied where no statistically significant difference was found between the treatments for the height and diameter growth variables $(p>0.85)$ and $(p>0.99)$ respectively, the product moment correlations were also applied from Pearson where a force of direct linear relationship was identified between the number of flowers, male and female with the number offruits ( $p>0.93$ ), achieving a production of 16.3 ton/ha. It was concluded that after producing S. sessiliflorum in the nursery the need to add amendments to the soil is reduced, however it is very important to select sites rich in organic content and carry out management practices such as cleaning, tutoring and pruning that are decisive for optimizing yields and productivity.

Keywords: Chocó, Colombia, Topiro, Phenology, Vuelta Mansa.

\section{Introducción}

El lulo es originario de zonas interandinas en América y se encuentra distribuido en centro y sur América; su cultivo se desarrolla bien entre los 1.600 y $2.500 \mathrm{~m}$ de altitud con temperaturas entre los $11^{\circ} \mathrm{C}$ y $25^{\circ} \mathrm{C}$; pertenece a la familia de las Solanaceas, produce frutos de pulpa verde, que por su color, sabor y valor nutritivo son muy apetecidos en los mercados nacionales y con amplias perspectivas para exportación a los mercados de Japón, Estados Unidos y comunidades europeas (Alemania) (Lobo y Medina 1999). Tiene importancia económica porque posee un excelente potencial a nivel industrial para la elaboración de néctar, mermeladas, helados, postres, jugos $\mathrm{y}$ dulces.

En Colombia el área dedicada a la producción de frutas en las últimas cuatro décadas pasó de $1,6 \%$ a $12,6 \%$ con un crecimiento sostenido hasta el año 2005 (legiscomex.com 2008). El lulo en la actualidad representa $0,1 \%$ de las exportaciones de frutas exóticas de Colombia (PROEXPORT Colombia 2008).

Para el Ministerio de Agricultura y Desarrollo Rural(MADR) (citado por Muñoz 2010), existían en Colombia 5.493 ha de lulo en el año 2005, sembradas sobre todo en Huila, Valle del Cauca, Boyacá y Nariño, con cerca del 58\% del área; el resto se encuentra distribuido en 17 departamentos más; sin embargo, estas cifras de área sembrada, corresponden a Solanum quitoense que es el lulo comercial en gran parte del país.

El departamento del Chocó reporta gran cantidad de especies vegetales con perspectiva de biocomercio y potencial para procesos de trasformación agroindustrial; dentro de estas resalta el lulo grande Solanum sessiliflorum o topiro que se comercializa como fruta fresca para la preparación de jugos, néctar, mermeladas, helados, postres y dulces entre otros. Tradicionalmente $S$. sessiliflorum o $S$. topiro es cultivado en huertos caseros bajo criterios del productor local, es decir, sin prácticas agronómicas que permitan identificar condiciones adecuadas de manejo, controlar plagas y enfermedades para optimizar su rendimiento productivo, situación que se con- 
vierte en una desventaja para la productividad en futuras iniciativas empresariales desarrolladas por el productor local. Asimismo, el desconocimiento de la aptitud de los suelos y su adaptabilidad a las condiciones agroclimáticas de la zona, es una limitante para su siembra a gran escala.

A pesar de que existe un paquete tecnológico amplio para $S$. quitoense y en la amazonia brasileña, colombiana y peruana, se puede encontrar un manual técnico para la cocona $S$. sessiliflorum Dunal, existe poca información sobre la fenología, crecimiento y posible domesticación bajo las condiciones agroclimáticas del Chocó Biogeográfico. Este es un recurso alimenticio económico para los pobladores de la región; en los últimos años se implementaron iniciativas empresariales donde demandan gran cantidad del producto para la fabricación de helados, salsas dulces y jugos.

Por la importancia de esta especie es necesario implementar alternativas que garanticen el recurso para las nuevas generaciones y que a su vez permitan potenciar el aprovechamiento sostenible de las mismas. Es por ello que la presente investigación tiene como objeto evaluar las fenología y crecimiento del Solanum sessiliflorum Dunal o Topiro bajo condiciones del departamento del Chocó, con el fin de aporta elementos para su manejo técnico además de generar información para la toma de decisión frente a la necesidad de obtener un mejor rendimiento y productividad de la especie.

\section{Materiales y métodos}

Área de estudio. Corresponde a la vereda Vuelta Mansa, ubicada geográficamente a $5^{\circ} 33^{\text {' }}$ 4.7" de latitud norte y $76^{\circ} 38^{\prime} 13$ " de longitud oeste, pertenece al corregimiento de Samurindo, municipio del Atrato, con una extensión superficial de $725 \mathrm{~km}^{2}$, temperatura promedio $28^{\circ} \mathrm{C}$, precipitación promedio anual de $8.000 \mathrm{~mm}$ y una altura de $32 \mathrm{msnm}$. Corresponde a las formaciones de zonas de vida, bosque pluvial Tropical (bp-T) y bosque muy húmedo Tropical (bmh-T) (EOT 2004). Limita al norte con el municipio de Quibdó, al sur con el municipio de Cértegui, al oriente con el municipio de Lloró y al occidente con Río Quito (PDM del Atrato 2009-2011) (Figura 1).

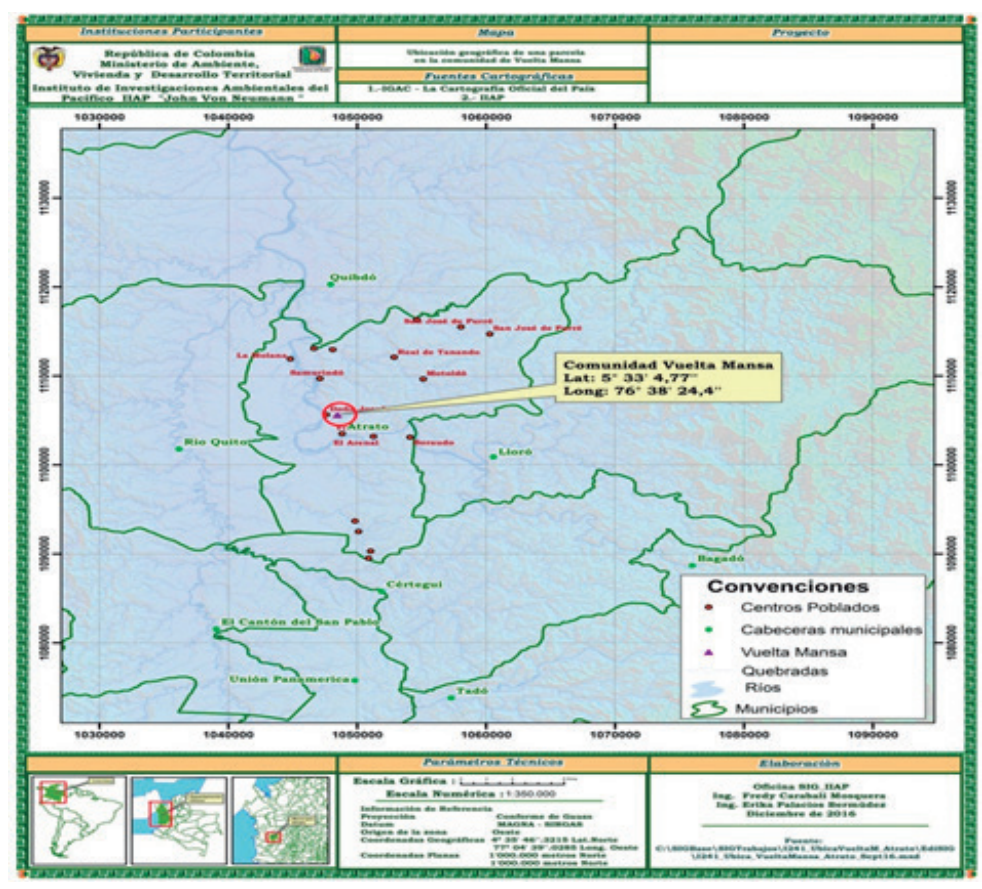

Figura 1. Localización de la vereda Vuelta Mansa, municipio de Atrato, Chocó, Colombia. 
Bioetnia Volumen 14, 2017

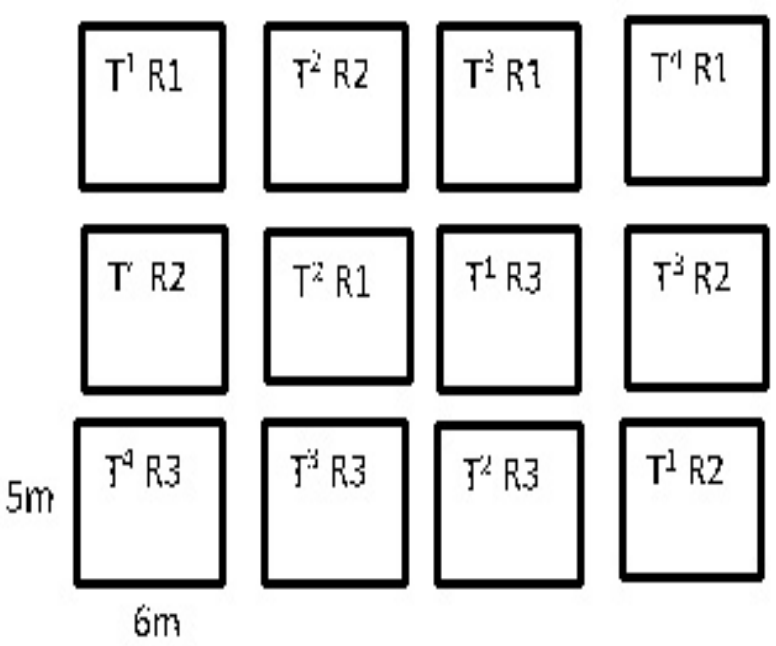

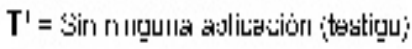

$$
\begin{aligned}
& T^{2}=\text { Aflicar } 209 \text { a a da }
\end{aligned}
$$

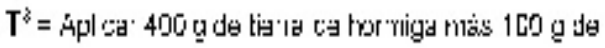

\section{Figura 2. Diseño completamente al azar con los tratamientos implementados para} evaluación de las diferentes variables estudiadas.

Método. Material experimental. Se seleccionaron frutos de S. sessiliflorum (lulo chocoano) de mejor tamaño y condiciones fenotípicas y se extrajeron las semillas que fueron sometidas a procesos de germinación. Luego las plántulas se trasplantaron a bolsas donde se dejaron hasta obtener una altura de $15 \mathrm{~cm}$.

Diseño de experimento y tratamiento. Para el estudio se empleó un diseño completamente al azar con cuatro tratamientos, tres repeticiones y cuatro por réplica. El tratamiento $1\left(\mathrm{~T}^{1}\right)$ consistió en la siembra de las plántulas directamente en el suelo; este tratamiento recibió el nombre de testigo. En el $\mathrm{T}^{2}$ se realizó la siembra de las plántulas con aplicación de $200 \mathrm{~g}$ de cal agrícola; en el $\mathrm{T}^{3}$ se le aplicó al suelo $100 \mathrm{~g}$ de cal agrícola y 400 g de tierra de hormiga; y finalmente en el $\mathrm{T}^{4}$ se aplicó $400 \mathrm{~g}$ de tierra de hormiga. Una vez definidos los tratamientos, se delimitó y adecuó un área de $920 \mathrm{~m}^{2}$ en la que se establecieron 12 parcelas de $5 \mathrm{~m}$ x $6 \mathrm{~m}\left(30 \mathrm{~m}^{2}\right)$ con distancias de un metro entre ellas. Por parcela se establecieron 16 plántulas de $S$. sessiliflorum a 1.5 m entre surco y 2 m entre planta, para un total 192 plántulas en estudio (Figura 2). Todo el material vegetal fue producido técnicamente en condiciones de vivero y llevado al sitio definitivo cuando las plántulas tenían una altura aproximadas de $15 \mathrm{~cm}$.

Variables evaluadas. Del total de plántulas sembradas se tomaron cuatro réplicas al azar durante la fase de crecimiento para determinar los aspectos como altura de la planta $(\mathrm{cm})$, diámetro $(\mathrm{cm})$, número de flores masculinas y femeninas, número de frutos, peso neto de la pulpa $(\mathrm{g}) \mathrm{y}$ rendimiento productivo por hectárea. Además, se registró el tiempo de germinación, floración y fructificación. La toma de datos se realizó de la siguiente manera:

Altura de la planta. Se midió cada quince días con un metro el incremento en altura, medida tomada desde la base de la planta hasta la yema apical del tallo principal.

Diámetro del tallo. Se registró el incremento en diámetro de la planta cada quince días con un Piederey digital, medida tomada en la mitad del tallo principal.

Número de flores por inflorescencia, flores masculinas y femeninas. Se registró la cantidad 
de flores por inflorescencias además de identificar cuáles correspondían a femeninas y masculinas.

Número de frutos cosechados por planta en cada tratamiento. Esta variable se tomó a partir del inicio de cosecha; todos los frutos cosechados se contabilizaron, igual que los caídos sanos y frutos caídos enfermos.

Peso de fruto. se usaron balanzas con precisión de 0,1 gramo, se pesaron frutos de diferentes tamaños.

Peso cáscara. La fruta se peló y se pesó en balanzas con precisión de 0,1 gramo.

Peso de semillas. Luego la fruta se licuó por poco tiempo y se pasó por un colador, el jugo se utilizó para las pruebas químicas. Las semillas se lavaron, secaron y pesaron.

Peso pulpa. Corresponde al cálculo de peso de fruta menos el peso de la cáscara y menos el de la semilla.

Porcentaje neto de pulpa. Resultado que se obtuvo de dividir el peso de pulpa entre el peso inicial de fruta por 100.

Rendimiento productivo por hectárea. Se realizó el cálculo a partir de la cantidad y peso de los frutos por cada planta partiendo de una densidad de siembra de 2.500 plantas por hectárea.

Días de inicio de floración. Se evaluó el tiempo transcurrido entre la siembra y cuando el 50\% de las plantas de cada parcela presentaron flores abiertas.

Días de presencia de frutos. Se evaluó el tiem- po transcurrido entre la siembra hasta cuando el $50 \%$ de las plantas de cada parcela presentaron frutos formados.

Días de cosecha. Se evaluó el tiempo transcurrido entre la siembra y cuando el $50 \%$ de plantas de cada parcela presentó frutos en madurez de cosecha.

Análisis de la información. Toda la información fue organizada y tabulada en bases de datos de Excel. Una vez tabulada la información y comprobados los supuestos de normalidad, se aplicó una prueba de ANOVA simple para comparar el efecto de los tratamientos sobre la altura y diámetro de las plantas. Además, se ejecutó un análisis de correlación, para determinar si existe relación entre el tipo de flor y el número de frutos obtenidos en cada tratamiento. Todos los análisis se realizaron con el paquete estadístico Statgraphics Centurion versión 2009 y se consideró el valor de $\mathrm{p}<0.05$ para determinar significancia estadística.

\section{Resultados y discusión}

Altura de la planta. La altura total promedio entre los tratamientos varía de $135 \mathrm{~cm}$ hasta 140 cm a los 210 días de sembrados, lo que evidencia un promedio de crecimiento mensual de 20 $\mathrm{cm}$ para la especie. En el ensayo no se encontró diferencia estadísticamente significativa $(\mathrm{p}>0.85)$ entre los tratamientos evaluados (Figura 3); sin embargo, la mayor elongación del tallo se observó

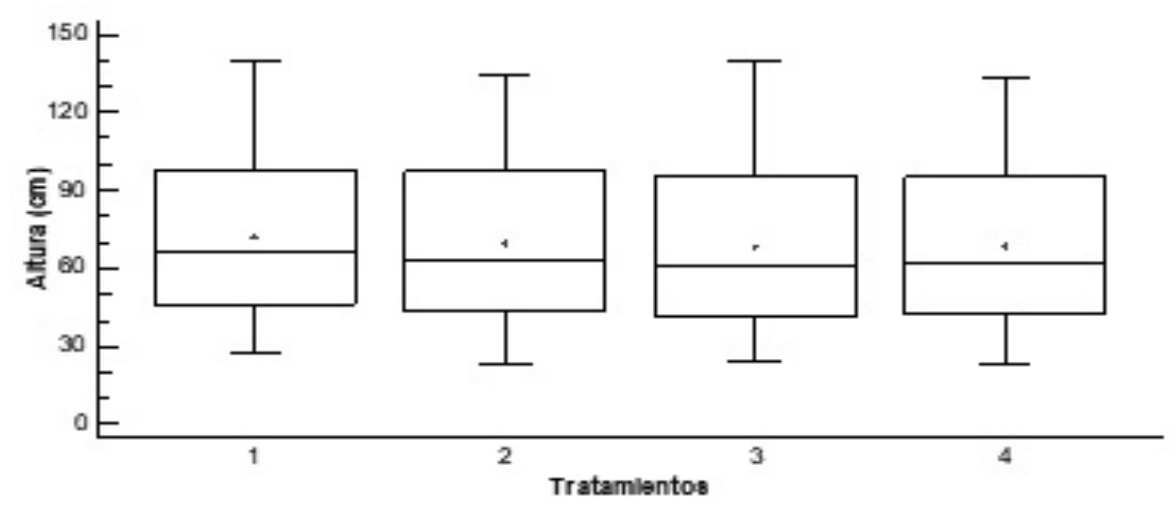

Figura 3. Distribución de las alturas registradas para S. sessiliflorum en los tratamientos evaluados. 


\section{Bioetnia Volumen 14, 2017}

en los tratamientos $\mathrm{T}^{1}$ ( $\sin$ sustrato) $\mathrm{y}^{3}$ (tierra de hormiga) ambos con $140 \mathrm{~cm}$; los resultados son diferentes a los reportados por Endara (2002) donde se observó que las plántulas de $S$. sessiliflorum alcanzaron alturas promedias de $96,65 \mathrm{~cm}$ a los 324 días de establecimiento del cultivo. Autores como da Silva e Izquierdo (1998) describen a la especie como un arbusto herbáceo de 1 a $2 \mathrm{~m}$ de altura, erecto y ramificado, características que están dentro del rango de crecimiento obtenido en este estudio.

La altura es de gran relevancia en el manejo de planta porque permite definir o ajustar protocolos de manejo específico para la especie bajo condiciones agroclimáticas de la zona, pues la plantas de mayor altura son más susceptibles a problemas de volcamientos asociados con el peso excesivo de los frutos y las condiciones de humedad del suelo; por otro lado la baja altura y la falta de poda crean microclimas que favorecen la presencia de enfermedades fungosas que afectarían la planta y la productividad del cultivo.

Diámetro del tallo. No se encontraron diferencia estadísticamente significativa $(\mathrm{p}>0,99$, Tabla 1) para los tratamientos en relación con el diámetro del tallo. La Figura 4 muestra el rango de distribución del diámetro para la especie que oscila entre 0,8 y 4,8 a los 210 días para los tratamientos $\mathrm{T}^{4} \mathrm{y} \mathrm{T}^{3}$ siendo estos los que reportaran los datos más elevados; no obstante, estos valores son muy similares a lo informado por los $\mathrm{T}^{1}$ que oscila entre 0,8 y 4,5 en igual tiempo de medición, siendo este el menor rango reportado. Los resultados son concordante con los obtenidos por Endara (2002) quien reportó para la especie, diámetros del tallo que variaron de 0,84

Tabla 1. ANOVA para diámetro por tratamiento

\begin{tabular}{lcrccc}
\hline Fuente & Suma de cuadrados & GI & Cuadrado medio & Razón-F & $\mathbf{p}$ \\
\hline Entre grupos & 0,185089 & 3 & 0,0616964 & 0,04 & 0,9900 \\
Intragrupos & 534,968 & 332 & 1,61135 & & \\
Total & 535,153 & 335 & & & \\
\hline
\end{tabular}

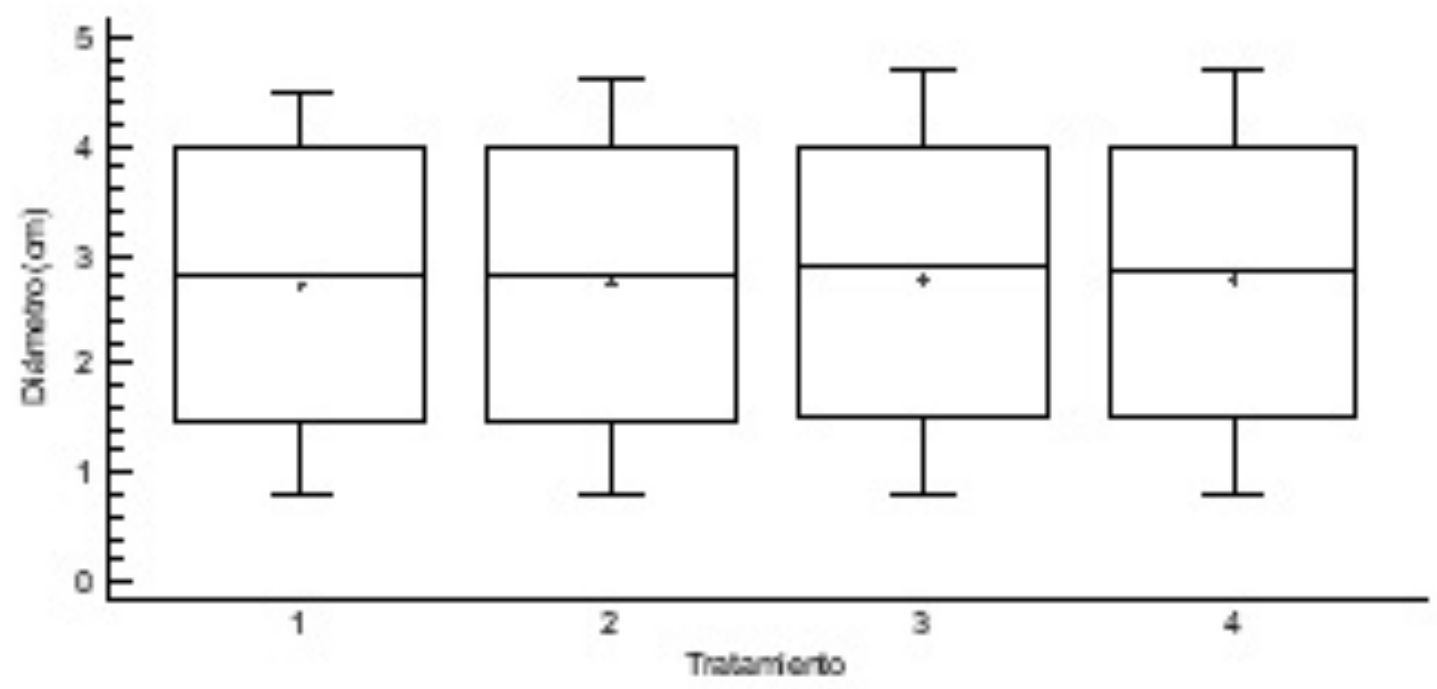

Figura 4. Distribución del diámetro del tallo registrado para S. sessiliflorum en los tratamientos evaluados. 
cm a los 79 días, a 6,02 cm a los 324 días; sin embargo, los reportes específicos en los 259 días de sembrados fueron en promedio de $3,56 \mathrm{~cm}$, siendo inferior a los reportados en este estudio y a su vez sugiere que la especie alcanza una mayor vigorosidad bajo las condiciones agroclimáticas de la zona, aspecto importante porque el diámetro es una variable trascendental por el transporte de nutriente a la parte superior de la planta y el soportar las ramas, hojas y frutos. En relación con la especie estudiada, su adaptabilidad a las condiciones climáticas de la zona permitió un incremento en los rangos aceptables para un desarrollo adecuado de la planta en los diferentes tratamientos evaluados, ratificando el peso que tiene la preferencia ecológica y relaciones evolutivas en el momento de seleccionar ambientes para la producción de las especies, pues estos factores inciden en la expresión de su máximo potencial de crecimiento y desarrollo (Ardila et al. 2011).

Número deflores. El número de flores muestra una correlación positiva perfecta entre el tipo de flor y la presencia de frutos ( $p>0,93)$; sin embar- go, se observa que el número de inflorescencia por planta es superior para los tratamientos $\mathrm{T}^{1} \mathrm{y}$ $\mathrm{T}^{2}$ con un valor promedio de 10 inflorescencia, datos que son superiores a los reportados para los tratamientos $\mathrm{T}^{3}$ y $\mathrm{T}^{4}$ donde solo se observaron 9 y 8 respectivamente (Tabla 2 ).

Los datos de este estudio superan los informados por León-Fuentes y Navarrete-Flores (2014), quien obtuvo promedio 5,49 flores por inflorescencia; esta variable es de gran importancia porque se observó una relación directamente proporcional entre el tipo y número de flores con la cantidad de frutos.

La Tabla 3 muestra el número de flores por tratamiento y la cantidad de flores masculinas y femeninas en cada inflorescencia evidenciando la condición de planta hermafrodita y la correlación antes descrita

$$
(f+m=F)
$$

donde:

$\mathrm{f}=$ flores femeninas, $\mathrm{m}=$ flores masculinas,

$\mathrm{F}=$ frutos

Tabla 2. Número de inflorescencia por tratamiento registrado en el corregimiento de Vuelta Mansa, municipio de Atrato, Chocó

\begin{tabular}{|c|c|c|c|c|}
\hline \multirow[t]{2}{*}{ Tratamiento } & \multicolumn{2}{|c|}{ Número de } & \multicolumn{2}{|c|}{ Flores } \\
\hline & cencia/planta & flores/inflorescencia & masculinas/planta & femeninas/planta \\
\hline $\mathrm{T}^{1}$ Sin testigo & 10 & 51 & 35 & 16 \\
\hline $\mathrm{T}^{2} 200 \mathrm{~g}$ cal agrícola & 10 & 54 & 38 & 16 \\
\hline \multicolumn{5}{|l|}{$\mathrm{T}^{3} 100 \mathrm{~g}$ de cal +} \\
\hline $400 \mathrm{~g}$ tierra de hormiga & 9 & 46 & 32 & 14 \\
\hline $\mathrm{T}^{4} 400 \mathrm{~g}$ tierra de hormiga & 8 & 36 & 24 & 12 \\
\hline Promedio total & 9,7 & 46,7 & 32 & 14,5 \\
\hline
\end{tabular}

Tabla 3. ANOVA para altura por tratamiento

\begin{tabular}{lcrccc}
\hline Fuente & Suma de cuadrados & GI & Cuadrado medio & Razón-F & p \\
\hline Entre grupos & 793,366 & 3 & 264,455 & 0,26 & 0,8572 \\
Intragrupos & 343,276, & 332 & 1033,96 & & \\
Total & 344,069, & 335 & & & \\
\hline
\end{tabular}




\section{Bioetnia Volumen 14, 2017}

En este sentido, se reportó un promedio de 46 flores por inflorescencia para todos los tratamientos de los cuales $68 \%$ (32) corresponden a flores masculinas y $32 \%(14,5)$ son flores femeninas, es decir que se presenta una proporción 2 a 1; los datos guardan gran relevancia porque bajo condiciones normales solo las flores femeninas se convertirán en potenciales frutos; sin embargo son las flores masculinas las encargadas de disponer del polen que se requiere para asegurar la fecundación de los óvulos de la flor femenina, procesos adelantados por polinización asistida $\mathrm{o}$ natural (anemófila o entomófila) haciendo posible la producción de frutos.

Número de frutos por planta. Los valores no presentan diferencia estadísticamente significativa $(\mathrm{p}>0,99)$ para los tratamientos evaluados. En la Figura 5 se presentan los resultados del número de frutos por planta para la variedad de lulo grande $(S$. sessiliflorum o topiro), donde el $\mathrm{T}^{1} \mathrm{y}$ el $\mathrm{T}^{2}$ presentaron los mejores promedios de frutos por planta, ambos con 16; el $\mathrm{T}^{3}$ mostró resultados menores (14) pero muy cercanos a los reportados por los anteriores tratamientos; el resultado más bajo se observó en el $\mathrm{T}^{4}$ con 12 frutos en promedio por planta. La especie reportó un promedio de 14,5 frutos por planta para todos los tratamientos; sin

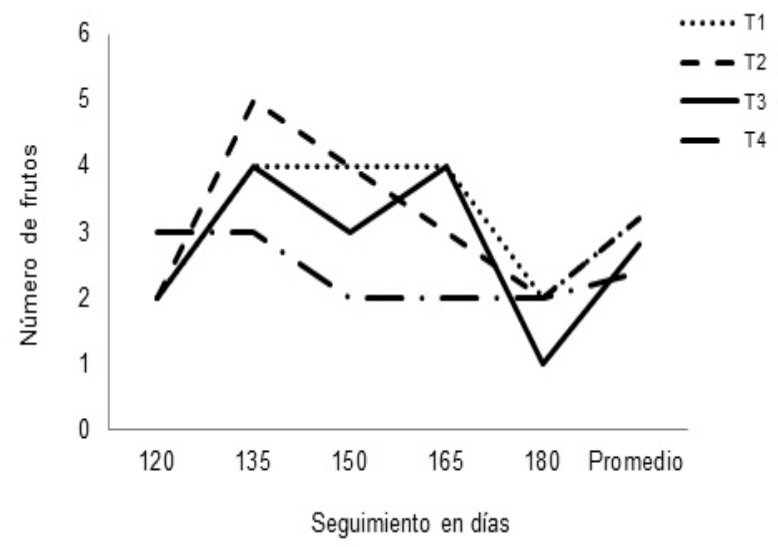

Figura 5. Seguimiento quincenal de la presencia de frutos promedio por tratamientos en plantas de lulo grande Solanum sessiliflorum o topiro sembradas en la vereda Vuelta Mansa, municipio de Atrato, Chocó. embargo, la poca diferencia observada entre los tratamientos podría estar asociada con el manejo dado a las plántulas en vivero y el aporte de materia orgánica contenido en el sospedón en el momento del trasplante a sitio definitivo. Este aspecto pudo haber sido más determinante para el desarrollo de la planta que las aplicaciones adicionales porque en todo momento el $\mathrm{T}^{1}$ siempre mostró resultados similares a los demás tratamientos evaluados.

Peso del fruto. La Tabla 4 muestra los datos de S. sessiliflorum o Topiro sembrada en condiciones agroclimáticas del Chocó, comparados con datos reportados para algunas especies en otros estudios realizados por distintos autores.

Los resultados muestran la gran diferencia que existe entre el peso promedio del fruto en gramos de $S$. sessiliflorum en relación con las otras especies, lo que se atribuye posiblemente con las características intrínsecas de la planta y con su buena adaptabilidad a las condiciones climáticas de la zona.

El porcentaje de pulpa neta varió para toda las especies de $58 \%$ a $67 \%$, sin embargo no se encontró una relación entre el peso del fruto y el peso neto de la pulpa, aspecto de gran importancia porque el tamaño y peso del fruto son las característica más relevantes para todos los actores de la cadena del lulo (Muñoz 2010), porque su uso en procesos de trasformación agroindustrial está relacionado con las características del endocarpio y mesocarpio de la fruta, no obstante $S$. sessiliflorum o topiro del Chocó, a pesar de estar constituido por $65 \%$ de pulpa y 35\% de cascara y semillas, reportó 227,5 g de pulpa, mientras que para $S$. solanum $s p$. y $S$. sessiliflorum Cocona (honduras) los valores son de 70 g y 122,4 g respectivamente; contrario a lo anterior, $S$. quitoense que es la variedad más comercial del país, tan solo reportó $55 \mathrm{~g}$.

De igual manera, la diferencia tan representativa en el peso de la pulpa es un factor de gran importancia para el desarrollo de procesos de agroindustria; este aspecto es concordante con algunos productores que manifiestan que los 
Tabla 4. Comparación del peso del fruto de S. sessiliflorum del Chocó con datos reportados para otras especies en otros estudios realizados

\begin{tabular}{|c|c|c|c|c|c|c|}
\hline \multirow[t]{2}{*}{ Especie } & \multicolumn{5}{|c|}{ Peso promedio de } & \multirow[b]{2}{*}{ Fuente } \\
\hline & fruto $(g)$ & cáscara (g) & semilla (g) & de pulpa (g) & $\%$ de pulpa & \\
\hline \multicolumn{7}{|c|}{ Solanum sessiliflorum } \\
\hline o topiro Chocó & 350 & 43,75 & 78,75 & 227,5 & $65 \%$ & Este estudio \\
\hline Solanum quitoense & 90 & 5 & 30 & 55 & $61 \%$ & Este estudio \\
\hline \multicolumn{7}{|l|}{ Solanum sessiliflorum } \\
\hline cocona (Honduras) & 181 & 2,91 & 48,81 & 122,42 & $67 \%$ & Endara (2002) \\
\hline Solanum sp. & 120 & 28 & 23 & 70 & $58 \%$ & Muñoz 2010 \\
\hline
\end{tabular}

Tabla 5. Distancia y densidad del ensayo vs. distancia y densidad sugerida luego de los resultados obtenidos para todos los tratamientos evaluados de $S$. sessiliflorum

\begin{tabular}{lccccc}
\hline Especies & $\begin{array}{c}\text { Distancia de } \\
\text { siembra }(\mathbf{m})\end{array}$ & $\begin{array}{c}\text { Densidad de } \\
\text { siembra }\end{array}$ & $\begin{array}{c}\mathbf{N}^{\circ} \text { promedio de } \\
\text { frutos por planta }\end{array}$ & $\begin{array}{r}\text { Peso promedio } \\
\text { en kg }\end{array}$ & $\begin{array}{c}\text { Rendimiento } \\
\text { (ton/ha) }\end{array}$ \\
\hline S. sessiliflorum & $1,5 \times 2,0$ & 3.333 & 14 & 0,35 & 16,3 \\
S. sessiliflorum & $2,5 \times 2,5$ & 1.600 & 14 & 0,35 & 7,8 \\
\hline
\end{tabular}

materiales que están por encima de $100 \mathrm{~g} /$ fruto son aceptados por el mercado de consumo fresco (Muñoz 2010).

Rendimiento productivo por hectárea. Los resultados fueron extrapolados a una hectárea para la cual se calculó una densidad de siembra de 3.333 árboles por hectárea-plántulas, distanciadas en $1,5 \mathrm{~m}$ x $2 \mathrm{~m}$, obteniendo en promedio 14 frutos por planta con pesos promedios de $350 \mathrm{~g}$, valor equivalente a $4,9 \mathrm{~kg}$ por planta que permitieron obtener 24,5 ton/ha; sin embargo, las observaciones de campo permiten deducir que para el cultivo bajo las condiciones de humedad de la zona es pertinente aumentar la distancia de siembra, seleccionar sitios con presencia de materia orgánica lo que reduciría el riesgo de presencia de enfermedades asociadas con la humedad y bajo contenido nutricional del suelo (Tabla 5); en relación con esto da Silva e Izquierdo (1998) y Villachica (1996). manifiestan que el rendimiento por hectárea depende de la etnovariedad cultivada, de la fertilidad del suelo, del suministro hídrico, de la densidad de plantas y del tipo de manejo adoptado. Algunos datos experimentales demuestran cómo esta combinación de factores se traduce en productividad.

Desarrollo y estados fenológicos. La Tabla 6 muestra los resultados de las etapas fenológicas de la especie bajo condiciones naturales de la zona de estudio; esta evidencia que el período trascurrido desde la germinación de semillas hasta la cosecha de frutos maduros oscila entre 224 y 260 días aproximadamente. No obstante, el ciclo biológico cambia con el genotipo y con los factores ambientales, esto quiere decir, que las plantas del mismo genotipo sembradas bajo diferentes condiciones climáticas pueden presentar diferentes estados de desarrollo después de transcurrido el mismo tiempo cronológico, por esto el uso de escalas fenológicas permite realizar prácticas culturales en etapas de desarrollo determinado (García 2003, citado por Muñoz 2010). 
Bioetnia Volumen 14, 2017

Tabla 6. Tiempos de desarrollo fenológico de S. sessiliflorum o topiro bajo condiciones de la zona de estudio

\begin{tabular}{lc}
\hline \multicolumn{1}{c}{ Etapa } & Tiempo en días \\
\hline Semilla a inicio de germinación & $6-12$ \\
Inicio de germinación a transporte a bolsas & 20 \\
Trasplante a bolsa a siembra en sitio definitivo & 30 \\
Siembra a presencia de yema floral & $105-120$ \\
Yema floral a apertura de flor & $5-8$ \\
Apertura de flor a formación de frutos & $8-10$ \\
Formación de frutos a iniciación de madurez & $30-40$ \\
Iniciación de madurez a madurez completa & 20 \\
\hline
\end{tabular}

Fuente: Moreno H. 2015 con base en lo planteado por García J 2003

\section{Conclusiones y recomendaciones}

Se concluyó que en $S$. sessiliflorum la producción de plántulas en viveros donde el llenado de bolsas se realice con sustratos de origen orgánico, tiene influencia en el desarrollo de la planta reduciendo la necesidad de adicionar enmiendas al suelo; no obstante es muy importante la selección de los sitios con buen contenido de materia orgánica y realizar prácticas de manejo como limpias, tutorado y poda que son determinantes para optimizar los rendimientos y productividad.

El crecimiento de la planta en tallo y altura fue relativamente similar en todos los tratamientos; estas variables son determinante en el desarrollo del cultivo porque a mayor altura aumenta la posibilidad de volcamiento de la planta, aspecto que está asociado con el peso excesivo de los frutos, además, se requiere de un tallo resistente y fuerte que soporte toda la parte superior que la planta (tallos superiores, hojas y frutos). Por otro lado, se evidencia una correlación muy fuerte entre la presencia de flores masculinas y femeninas con la presencia de frutos, a pesar de que las flores femeninas ocupan $30 \%$ del total de flores reportadas, estas se convirtieron en potenciales frutos.

Los frutos de $S$. sessiliflorum sembrado en el Chocó son los más grandes de todas las variedades de lulo reportadas; el peso neto es determinante en procesos de desarrollo agroindustrial siendo la especie promisoria para este tipo de usos.

Se recomienda realizar podas de formación y diseñar una estructura arquitectónica de la planta que sea ideal y balanceada para evitar el volcamiento y aumentar la productividad asociada con la humedad del suelo. Además, es pertinente avanzar a procesos de investigación para lograr aumentar la cantidad de flores femeninas dentro de cada inflorescencia lo que produciría un aumento de la productividad cercano a $50 \%$.

\section{Literatura citada}

Ardila G, Fisher G, Balaguera-López HE. Caracterización del crecimiento del fruto y producción de tres híbridos de tomate (Solanum lycopersicum L.) en tiempo fisiológico bajo invernadero. Rev Colomb Cienc Hortic. 5 (1): 44-56. doi: 10.17584/ rcch.2011v5i1.1252

Da Silva Filho DF, Izquierdo J. 1998. Cocona (Solanum sessiliflorum Dunal): cultivo y utilización. Caracas: Secretaría Pro-Tempore, Tratado de Cooperación Amazónica.

Endara EP. 2002. Estudio fenológico de la cocona (Solanum sessiliflorum Dunal). Tesis de grado. Honduras: Zamorano, Carrera de Ciencia y Producción Agropecuaria; 46 pp. Disponible en: https://bdigital.zamorano.edu/ bitstream/11036/2350/1/CPA-2002-T047.pdf

García J. 2003. Evaluación del crecimiento de dos ecotipos 


\section{Crecimiento y fenología de S. sessiliflorum. HL Moreno, SY Mosquera}

de lulo amazónico (Solanum sessiliflorum Dunal) bajo tres ambientes en el piedemonte amazónico del Caquetá. (Tesis de maestría). Bogotá: Universidad Nacional de Colombia; 63 pp.

Legiscomex. Sistema de inteligencia comercial. 2008. Frutas exóticas en Colombia. Inteligencia de mercados. Caracterización del sector. Bogotá: Legis. Disponible en: https://www.legiscomex.com/BancoMedios/Documentos $\% 20 \mathrm{PDF} /$ est col frutas exot 2.pdf

León-Fuentes JF, Navarrete-Flores JL. 2014. Evaluación de reguladores orgánicos de crecimiento para el engrose del fruto de naranjilla (Solanum quitoense Lam.). San Miguel de los Bancos-Pichincha. Tesis de grado. Quito: Facultad de Ciencias Agrícolas, Universidad Central del Ecuador; 88 pp. Disponible en: http://www.dspace.uce. edu.ec/bitstream/25000/3030/1/T-UCE-0004-91.pdf

Lobo M, Medina CI. 1999. Lulo o naranjilla (Solanum quitoense Lam), frutal andino con potencial de desarrollo. Bogotá: CORPOICA.

Municipio delAtrato. 2004. Esquema de ordenamiento territorial. Documento de diagnóstico. Alcaldía municipal 2004-2015. Una propuesta social donde cabemos todos. Disponible en: http://cdim.esap.edu.co/BancoMedios/ Documentos\%20PDF/atratochocoeot2004-2015.pdf
Muñoz LA. 2010. Evaluación agronómica de materiales de lulo Solanum sp., frutal de alto potencial para zonas tropicales. Palmira: Conecta rural. Disponible en: http://conectarural.org/sitio/material/evaluaci\%C3\%B3n-agron $\% \mathrm{C} 3 \% \mathrm{~B} 3$ mica-de-materiales-de-lulo-solanum-sp-frutal-de-alto-potencial-para-zonas

Plan de Desarrollo Municipio del Atrato 2008-2011. Una propuesta social: compromiso de todos. Disponible en: http://cdim.esap.edu.co/BancoMedios/Documentos\%20PDF/atrato\%20-\%20antioquia \%20-\%20 $\mathrm{pd} \% 20-\% 202008 \% 20-\% 202011 \% 20$ (pag\%2096\%20 $-\% 201.084 \% 20 \mathrm{~kb}) . \mathrm{pdf}$

PROEXPORT Colombia. 2008. Informe frutas exóticas, mermeladas y frutas deshidratadas. Bogotá: PROEXPORT.

Villachica H, de Carvalho JEU, Muller CH, Díaz SC, Almanza M. 1996. Frutales y hortalizas promisorios de la Amazonia. Lima: Tratado de Cooperación Amazónica, Secretaría Pro-Tempore. Disponible en: http:/www.bdpa.cnptia.embrapa.br/consulta/busca?$\underline{b}=$ pc\&id $=387185 \&$ biblioteca $=$ vazio\&busca $=$ autoria:\%22VILLACHICA \%20H.\%22\&qFacets=autoria:\%22VILLACHICA \%20H.\%22\&sort=\&pagina$\underline{\mathrm{cao}}=\mathrm{t} \&$ paginaAtual $=1$ 